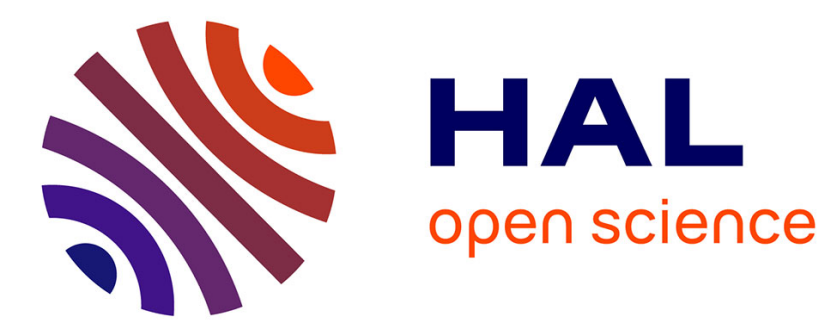

\title{
Le lexique de l'étonnement chez Appien. Quelques remarques
}

Philippe Torrens

\section{To cite this version:}

Philippe Torrens. Le lexique de l'étonnement chez Appien. Quelques remarques. KTÈMA Civilisations de l'Orient, de la Grèce et de Rome antiques, 2017, 42, pp.233-250. halshs-01670182

\section{HAL Id: halshs-01670182 \\ https://shs.hal.science/halshs-01670182}

Submitted on 21 Dec 2017

HAL is a multi-disciplinary open access archive for the deposit and dissemination of scientific research documents, whether they are published or not. The documents may come from teaching and research institutions in France or abroad, or from public or private research centers.
L'archive ouverte pluridisciplinaire HAL, est destinée au dépôt et à la diffusion de documents scientifiques de niveau recherche, publiés ou non, émanant des établissements d'enseignement et de recherche français ou étrangers, des laboratoires publics ou privés. 

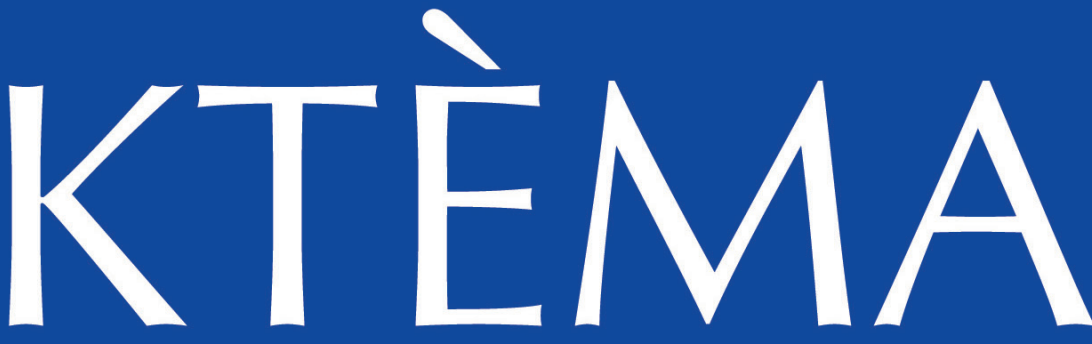

\section{CIVILISATIONS DE L'ORIENT, DE LA GRÈCE ET DE ROME ANTIQUES}

Edith Foster

Edmond LÉvY

Tim Roop

Cinzia Bearzot

\section{Michel Нumm}

David S. Levene

Estelle OudoT

Hans Kopp

Maciej JUNKIERT

Tobias Јоно

Christian WENDT

Oliver SCHELSKe

Dominique Lenfant

Neville MORLEY

Agnès Molinier Arbo

Michèle Coltelloni-Trannoy Philippe Torrens

Jean Ducat

Michel WoronofF

\section{Les interprétations de la défaite de 404}

Interpretations of Athen's defeat in the Peloponnesian war............................................................ 7

Thucydide, le premier interprète d'une défaite anormale .............................................................. 9

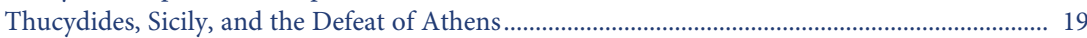

La $\sigma u \mu \varphi$ ód de la cité

La défaite d'Athènes (405-404 av. J.-C.) chez les orateurs attiques.............................................. 41

Rome, une «cité grecque» prise par les Hyperboréens.................................................................... 53

Rome Redeems Athens?

Livy, the Peloponnesian War, and the Conquest of Greece............................................................ 73

Ultime défaite d'Athènes ou sa plus belle victoire?

Stratégies rhétoriques autour de la bataille d'Aigos-Potamoi

dans le Panathénaïque d'Aelius Aristide

The Defeat of Athens in 404 BC in The Federalist ............................................................................ 97

Polish Reflections: The Reception of the Defeat of Athens in

the Works of Gottfried Ernst Groddeck and Joachim Lelewel.........................................................115

The Internal Commotion of Greek Culture: Jacob Burckhardt

on the Defeat of Athens in the Peloponnesian War.

Spree-Athen nach dem Untergang

Eduard Meyer zur Parallelität von Geschichte

Der Kampf um die Demokratie

Thukydides in Deutschland nach dem Ersten Weltkrieg

Défaite militaire et révolution antidémocratique

Le parallèle entre l'Athènes de 404 et la France de 1940

dans Les Oligarques de Jules Isaac.

Thucydides and the Historiography of Trauma.

\section{Le thauma dans l'historiographie grecque d'époque impériale}

Hérodien, Rome et le spectacle du pouvoir

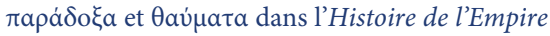

après la mort de Marc Aurèle

La place du thauma dans l'Histoire romaine de Cassius Dion............................................................219

Le lexique de l'étonnement chez Appien. Quelques remarques .......................................................23

\section{Varia}

Du caractère «mixte» du régime spartiate 251

L’image de la défaite dans l'Iliade. 


\author{
KTÈMA \\ CIVILISATIONS DE L'ORIENT, DE LA GRÈCE ET DE ROME ANTIQUES \\ Revue annuelle \\ Fondateurs : Edmond Frézouls † \\ Edmond LÉvY
}

Comité Directeur: Dominique Beyer, Bruno Bleckmann, Jean-François Bommelaer, Frédéric Colin, MireilleCorbier, GérardFreyburger, Jean Gascou, Jean-Georges Heintz, Michel Humbert, Anne Jacquemin, Stavros Lazaris, Dominique Lenfant, Edmond Lévy, Jean-Claude Margueron, Henriette Pavis d'Escurac, Laurent Pernot, Thierry Petit, Gérard SiEBERT

Rédaction:Edmond LÉvy

Dominique Beyer et Gérard Freyburger

Maquette et mise en page: Ersie LERIA

\title{
Éditeur
}

Presses universitaires de Strasbourg

5 allée du Général Rouvillois - CS50008

FR-67083 Strasbourg Cedex

Tél: (33) 0368856265

info.pus@unistra.fr

pus.unistra.fr

\section{Ventes au numéro}

En librairie ou en commande en ligne sur le site du Comptoir des presses d'universités: www.lcdpu.fr

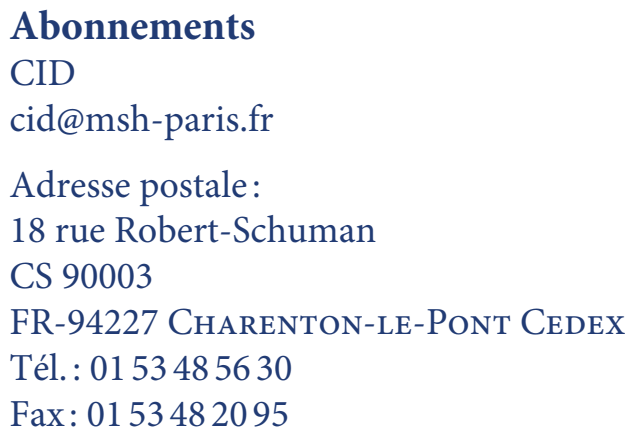




\title{
Le lexique de l'étonnement chez Appien
}

\author{
Quelques remarques
}

\begin{abstract}
RÉsumé-. En tant que narrateur, Appien n'exprime guère d'étonnement ( $\theta a \tilde{\mu} \mu \alpha)$ et, quand il le fait, cela ne concerne pas des problèmes généraux, mais se limite à des situations particulières. En revanche, il attribue volontiers aux personnages et aux collectivités des étonnements admiratifs, suscités en particulier par des

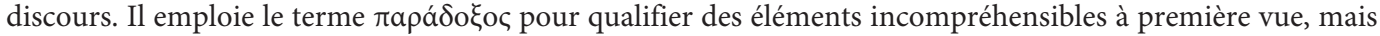
que le détail de son récit éclaire; c'est là un ressort du récit qui ne concerne jamais le narrateur. L'étonnement

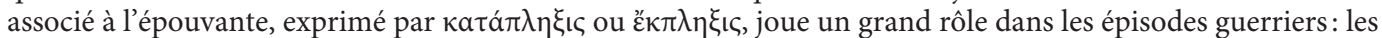
meilleurs stratèges œuvrent à le créer chez les ennemis et les combattants expérimentés savent s'en préserver. Pour les phénomènes surnaturels, Appien n'innove guère et il se contente de les couler dans la logique de son récit sans leur accorder un rôle prépondérant. S’il ne laisse que peu de place au $\theta a \tilde{u} \mu \alpha$ dans sa démarche d'historien de Rome, c'est parce que pour lui l'ascension de Rome est inscrite dans ses qualités fondamentales, qu'il définit dès sa Préface. Son récit illustre un schéma explicatif exposé dès le départ, ce qui laisse peu de place à l'interrogation du narrateur. En outre, ce schéma ne concerne que le passé et l'historien ne se projette sur aucun avenir.
\end{abstract}

Riassunto-. Come narratore, Appiano esprime raramente la propria sorpresa ( $\theta \alpha \tilde{v} \mu \alpha)$ e, se lo fa, ciò non riguarda dei problemi generali, ma soltanto delle situazioni particolari. Egli attribuisce invece volentieri ai personaggi o a gruppi di persone del racconto reazioni di sorpresa mista ad ammirazione, suscitata soprattutto

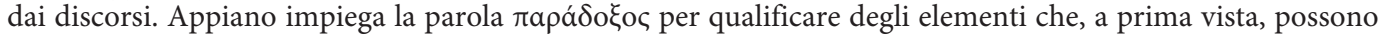
sembrare oscuri, ma che vengono chiariti nel corso del suo racconto dettagliato. Questa è tuttavia una strategia del narratore, ed egli non manifesta il suo punto di vista personale. La sorpresa associata allo spavento, espressa

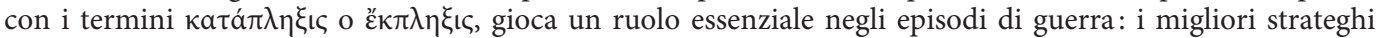
cercano sempre di provocarla nei nemici e i soldati esperti sanno esserne immuni. Per quel che riguarda $i$ fenomeni sovrannaturali, Appiano non si pone come innovatore, ma si limita ad integrarli alla logica del suo racconto senza attribuire loro un' importanza eccessiva. Nella sua qualità di storico delle vicende romane, egli da poco spazio al $\theta a \tilde{u} \mu \alpha$ : ciò deriva dal fatto che per lui l'ascesa di Roma è inscritta fin dall'inizio nelle sue qualità fondamentali che egli già definisce nella Prefazione. Il suo racconto illustra uno schema esplicativo stabilito dall'inizio, e ciò lascia poco spazio alle interrogazioni del narratore. Questo schema, inoltre, si limita al passato, e lo storico non fa nessuna proiezione sul futuro.

Chez Hérodote, $\theta$ aṽ $\mu$, l'étonnement interrogateur, possède une valeur heuristique et structurante $^{1}$. L'historien-enquêteur s'étonne face à de nombreuses réalités et il cherche des réponses qui puissent faire passer les $\theta a u ́ \mu a \tau a$, phénomènes provoquant l'étonnement et qu'une approche trop rapide pourrait amener à croire miraculeux, à l'état de réalités explicables. Cette

(1) Nous renvoyons notamment à HUNZINGER 1995. 
démarche trouve-t-elle encore un écho, après sept siècles d'écriture historique, chez un historien $\mathrm{du} \mathrm{II}^{\mathrm{e}}$ siècle après J.-C. comme Appien, dont la Préface énumérant les territoires qui composent l'empire romain et la forme géographique qu'il donne à l'organisation générale de son Histoire romaine pourraient faire attendre chez lui un intérêt ethnographique rappelant Hérodote? Notre première enquête portera sur les occurrences du mot $\theta \alpha \tilde{v} \mu \alpha$ et des termes apparentés $(\theta a v \mu a ́ \zeta \omega$,

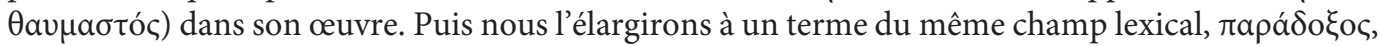
avant de nous intéresser à la présence dans la narration des réactions d'étonnement effrayé

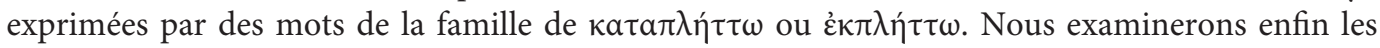
faits merveilleux ( $\tau \dot{\varepsilon} \rho \alpha \tau)$ retenus par Appien en analysant la façon dont il les traite et l'importance qu'ils revêtent dans son récit.

\section{OCCURRENCES DE @AYMA ET DE SES DÉRIVÉS CHEZ APPIEN}

Force est de constater d'emblée qu'elles ne sont pas très nombreuses: 41 en tout selon la Concordance publiée par E. Famerie ${ }^{2}$. Et un petit nombre seulement s'applique de façon nette au narrateur, avec diverses nuances. Ce sont ces occurrences-là que nous examinerons d'abord.

Ce ne sont pas des phénomènes, mais des comportements qui suscitent l'étonnement de l'historien. Ainsi, il s'étonne de voir que les peuples d'Illyrie voisins de la Cisalpine n'aient pas été soumis plus tôt par les Romains:

Je suis étonné de ce que beaucoup de grandes armées romaines, partant affronter les Gaulois ou les Ibères en franchissant les Alpes, aient négligé ces peuples et que même Caius César, qui a remporté de si grands succès dans ses guerres, n'en ait pas fini avec eux, lorsqu'il menait la guerre contre les Gaulois et que pendant dix ans il prenait ses quartiers d'hiver à proximité de cette contrée ${ }^{3}$.

Appien s'étonne aussi que, au plus fort du conflit civil autour de Tib. Gracchus, les Romains n'aient pas fait appel à un dictateur:

Il me paraît étonnant que <les Romains $>$ qui souvent, dans des situations si angoissantes, avaient assuré leur salut grâce à la dictature, n'aient alors même pas songé à nommer un dictateur et qu'une procédure qui avait été reconnue comme très utile pour leurs prédécesseurs ne soit ainsi pas même revenue au souvenir du peuple, ni à ce moment-là, ni plus tard ${ }^{4}$.

Appien, dans ces deux cas, s'étonne, mais ne fournit aucune explication aux comportements dont il signale l'apparente étrangeté. Il procède différemment quand il évoque la retraite volontaire du tout-puissant Sylla:

Il me parait étonnant ( $\theta \alpha \tilde{u} \mu \alpha)$ aussi de la part de Sylla qu'il ait été le premier et le seul jusqu'alors à avoir déposé un tel pouvoir, alors que rien ne l'y poussait, et ce, non pas en faveur de ses enfants, comme Ptolémée en Égypte, Ariobarzane en Cappadoce ou Séleucos en Syrie l'avaient fait, mais

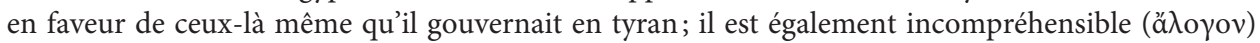
qu'après avoir conquis de force ce pouvoir en prenant de grands risques, une fois qu'il en fut maittre,

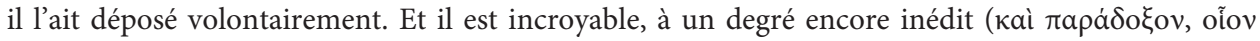

(2) FAMERIE 1995.

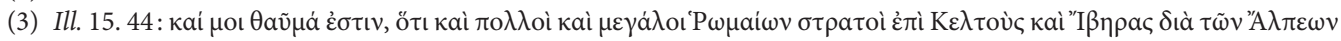

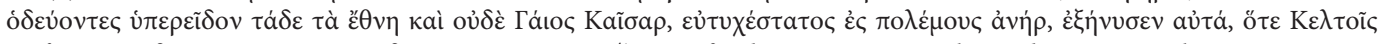

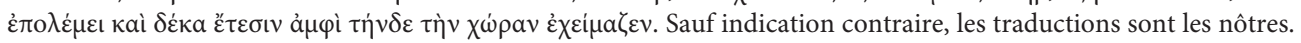

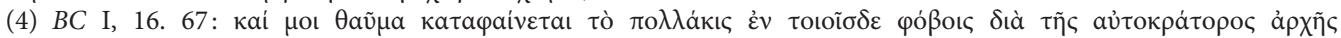

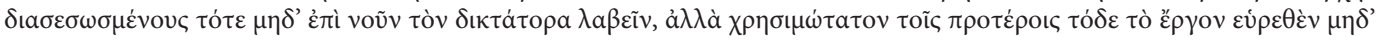

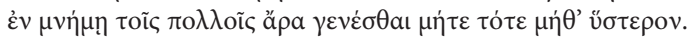




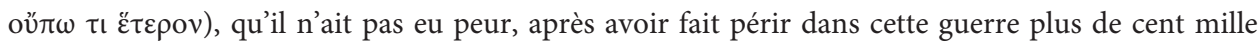
jeunes gens ${ }^{5} \ldots$

Contrairement aux cas précédents, Appien propose une explication en montrant peu après que cette décision est moins extraordinaire qu'il n'y paraît: Sylla pouvait se permettre de se retirer sans crainte parce que Rome et l'Italie étaient remplies des complices et bénéficiaires de ses actions et qu'il aurait pu, au besoin, les mobiliser très vite pour sa défense, qui se confondait avec celle de leurs acquis ${ }^{6}$. Tout se passe comme si l'historien relevait son récit en créant chez le lecteur, par l'association entre $\theta \alpha \tilde{v} \mu \alpha$, á̉ $\lambda o \gamma o \varsigma$ et $\pi \alpha \rho a ́ \delta o \xi o c$, une attente qu'il comblera un peu plus tard par une explication rationnelle.

Souvent aussi, l'étonnement du narrateur se double d'admiration devant ce qu'un comportement a d'inattendu, sans être cette fois incompréhensible. C'est dans ce sens qu'il relate comment, au cours de la troisième guerre Punique, les Carthaginois, se heurtant à l'intransigeance répétée des Romains, qui exigent d'eux l'évacuation de Carthage et leur installation à l'intérieur des terres, passent tout d'un coup de l'abattement à la résolution de résister: "Devant ce nouvel échec, étonnants furent leur changement d'attitude et leur audace: plutôt endurer tous les maux qu'abandonner leur cité $»^{7}$. L'historien signale un changement du même ordre, dont il fournit immédiatement une explication psychologique (la peur intense), à propos d'un proscrit qui, lorsqu'un soldat vint loger à proximité, «ne supporta même plus cette peur et passa de la lâcheté à une audace étonnante: il se rasa le crâne et dirigea une école en pleine Rome jusqu'aux accords de paix $»^{8}$.

Appien semble sensible aux points de rupture, qui induisent un changement radical ( $\mu \varepsilon \tau \alpha \beta$ o $\lambda$, $\mu \varepsilon \tau \alpha \beta a ́ \lambda \lambda(\omega)$. Ces revirements sont appelés par l'urgence désespérée de la situation, comparable à celle que connaît une armée en très grande difficulté: très souvent la conscience d'un danger extrême lui insuffle l'énergie qui lui permet de remporter la victoire sur un adversaire en meilleure posture $^{9}$. C'est ce qui, selon Appien, se passe à Pharsale ${ }^{10}$ et, plus encore, à Philippes ${ }^{11}$. Cette attention portée au sursaut d'audace dans une situation désespérée n'est que le cas particulier de l'intérêt plus général qu'accorde Appien aux changements du destin dans la vie des hommes ou des cités. Remarquons toutefois que c'est aux revirements positifs qu'il réserve le lexique de l'étonnement, en général admiratif, alors qu'il s'étend volontiers sur les grands revers de fortune qui font passer un homme de la puissance et de la richesse à l'état de misérable fugitif, ou une cité puissante et prospère à la ruine, sans marquer explicitement sa surprise ${ }^{12}$. L'émotion qui s'exprime

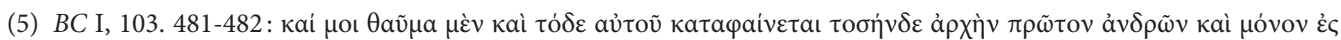

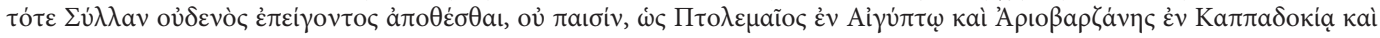

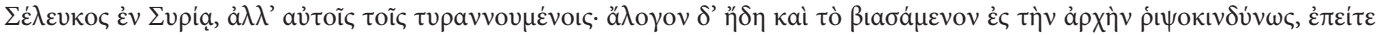

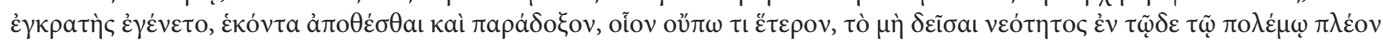

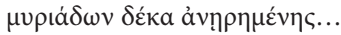

(6) BC I, 104. 489.

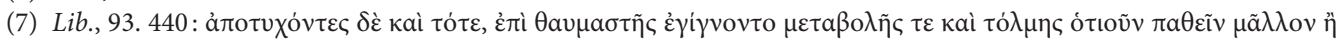

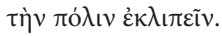

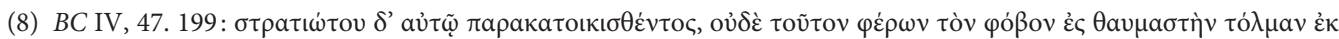

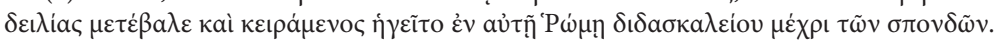

(9) Goldmann 1988, p. 56-60.

(10) BC II, 71. 297; 75. 312.

(11) BC IV, 127. 530; 128. 536.

(12) À propos par exemple de Carthage, Lib. 82. 384; 132. 628-629 (où Carthage, passée de la puissance à l'anéantissement, est rapprochée d'Ilion et des empires assyriens, perses et macédoniens; Scipion Émilien y exprime une crainte identique pour Rome); Lib. 27. 114 (Syphax); BC I, 61. 273 (Marius fugitif); BC III, 98. 407-408 (Decimus Brutus); $B C \mathrm{~V}, 126.522$ (Lépide); $B C$ II, 118. 498 (le cadavre de César, "qui peu auparavant était le maître de la terre et de la mer", est ramené chez lui, en brinquebalant, par trois esclaves). 
face à ces situations est alors la pitié (oĩ $\tau$ To $)^{13}$, voire les larmes comme celles que Scipion Émilien verse sur Carthage en flammes ${ }^{14}$.

L'étonnement du narrateur prend une nuance particulière dans toute la fin du livre III des Guerres civiles, qui rapporte les nombreux revirements de l'opinion et des dirigeants à Rome. À chaque fois, le narrateur feint de trouver ces changements étonnants alors que, de toute évidence, ils semblent s'installer comme une habitude. Cette répétition exprime une ironie manifeste. Appien raille ainsi la rapidité avec laquelle la population de Rome change de dispositions quand elle apprend la jonction de Lépide et d'Antoine en juillet 43 :

À l'annonce de ces événements à Rome, il se produisit de nouveau un changement étonnant et subit

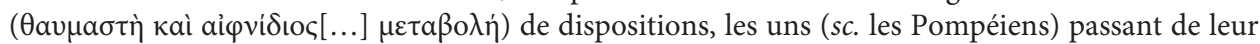
récente arrogance à la peur, les autres ( $s c$. les partisans d'Antoine) de la peur à la hardiesse ${ }^{15}$.

Dans la suite du texte, Appien poursuit cet effet en relatant un nouveau revirement des Romains quand Octave, en août 43, arrive à Rome et s'empare de la colline du Quirinal:

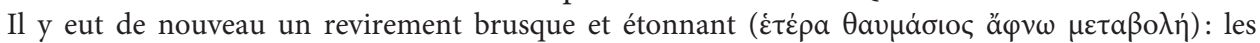
aristocrates coururent au-devant de lui et cherchèrent à lui parler; la masse du peuple y courait également et on voyait dans le bon ordre des troupes une disposition pacifique ${ }^{16}$.

Cet étonnement répété contribue à donner à tout le récit de la conquête de Rome par Octave en août 43 l'allure d'une farce, l'historien soulignant à l'envi les velléités et les rodomontades de la population romaine et de ses dirigeants - et en premier lieu, de Cicéron ${ }^{17}$. Tels sont les passages, assez peu nombreux en définitive, où Appien, en son nom propre de narrateur, exprime nettement son étonnement.

Il existe par ailleurs de plus nombreuses occurrences où cet étonnement, mêlé d'admiration, est attribué à des personnages ou à des collectivités, par exemple devant les qualités manifestées par certains hommes politiques. Tel est le sentiment de la population romaine écoutant le discours que Brutus lui adresse après le meurtre de César:

Tandis que Brutus tenait ces propos, tous ses auditeurs, en l'écoutant encore et en se dispersant, les approuvaient en les estimant très justes; ils admiraient ces hommes pour leur sang-froid et surtout pour leur amour du peuple, se mettaient à éprouver de la sympathie pour eux et s'apprêtaient à agir le lendemain à leurs côtés ${ }^{18}$.

C’est aussi à l'admiration pour César qu'Antoine pousse le peuple romain lors des funérailles du dictateur:

énumérant en même temps d'une voix précipitée ses guerres, ses batailles, ses victoires, les peuples qu'il avait adjoints à sa patrie, tout le butin qu'il avait envoyé, exaltant ce qu'avait d'extraordinaire

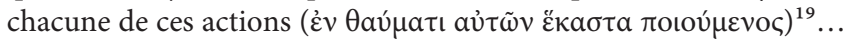

(13) Lib. 82. 384.

(14) Lib. 132. 628.

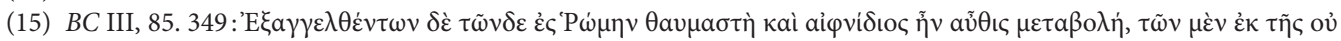

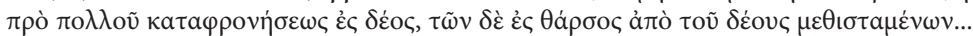

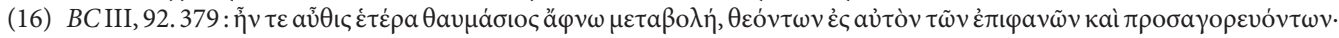

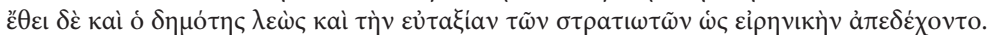

(17) BC III, 82-93 passim. Dans ce contexte, Appien exprime un étonnement narquois de façon moins directe quand il montre, quelques mois plus tôt, après la victoire surévaluée de Forum Gallorum, fin avril 43, les pompéiens de Rome, restés

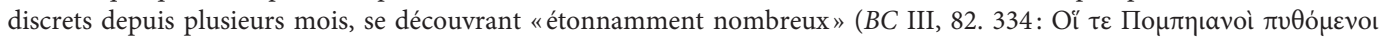

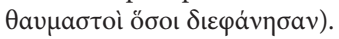

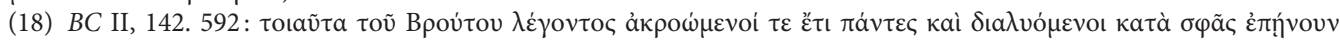

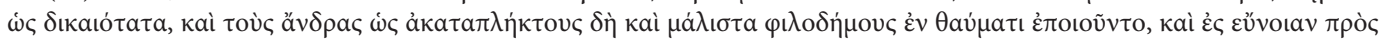

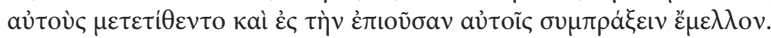

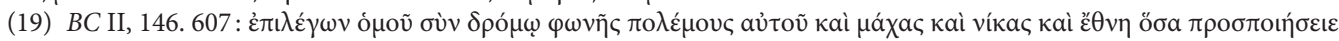

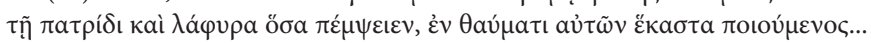


Un étonnement mêlé d'admiration est enfin ce qu'éprouve Octave quand, après sa victoire à Pérouse, il a écouté les nobles paroles de Lucius Antonius vaincu ${ }^{20}$. Les exemples précédents montrent que, souvent, l'étonnement admiratif est provoqué par des paroles, qui sont elles-mêmes sources d'actions: les auditeurs de Brutus s'apprêtent à agir avec lui, ceux d'Antoine ne vont pas tarder à incendier les maisons des meurtriers de César et à brûler le corps du dictateur sur le forum; Octave attribue une fonction importante à Lucius Antonius ${ }^{21}$. Les paroles sont d'ailleurs nécessaires, même quand c'est une démarche qui provoque l'étonnement: Coriolan est admiratif devant l'arrivée des femmes romaines qui ont l'audace de se rendre en délégation auprès de lui ${ }^{22}$, mais il faut toutes les supplications de sa mère pour qu'il change de position.

L'étonnement peut enfin se présenter pour ainsi dire à l'état pur, comme une réaction de surprise face à un élément inattendu, tel le retrait du lieutenant de Sextus Pompée, Ménodoros, et de sa flotte pour narguer les troupes d'Octave qui les croient échoués sur des hauts-fonds: « $<$ Menodoros $>$ fit marche arrière tout en riant; tout en s'étonnant, l'armée de César était envahie par la désolation $»^{23}$.

Enfin, le passage suivant montre un emploi rare chez Appien de $\theta$ aṽ $\mu \alpha$ au sens de "chose étonnante", seul exemple où il associe, implicitement toutefois, le mot au sens de la vue. L'épisode évoque l'armée d'Octave durant la guerre contre Sextus Pompée: "Alors qu'il était encore en train d'établir son camp, Pompée s'avança avec une flotte considérable, chose stupéfiante et inattendue

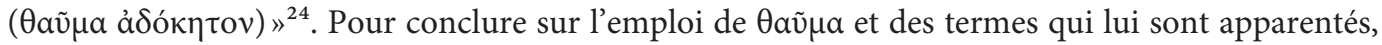
on remarquera qu'aucune de leurs occurrences ne porte sur un problème général qu'il faudrait élucider. Ainsi, nulle part dans sa Préface, Appien ne s'étonne de l'existence même des empires, ni même de l'extension exceptionnelle de l'empire romain, qu'il se limite à constater et à souligner par comparaison aux empires précédents. Ses étonnements sont suscités par des problèmes particuliers tels que l'abdication de Sylla ou la tardive conquête de l'Illyrie, ou par des épisodes précis de la narration, par rapport auxquels il prend parfois une distance ironique. Ils sont fréquemment en relation avec le changement et la rapidité. Ni chez le narrateur, ni chez les personnages, l'étonnement ne débouche sur une recherche d'explication: cette dernière est généralement fournie par le contexte immédiat. L'étonnement reste sans réponse seulement lorsque Appien se demande pourquoi les Romains, lors de la crise de Tib. Gracchus, n'ont pas choisi de nommer un dictateur, et quand il ne s'explique pas que les Romains n'aient pas soumis plus tôt l'Illyrie ${ }^{25}$. Par ailleurs, $\theta$ aṽ $\mu \alpha$ et les mots qui lui sont apparentés ne sont que rarement associés au sens de la vue ${ }^{26}$ et, pour ce qui est de l'ouïe, ne s'appliquent jamais à des bruits inattendus ou remarquables, mais seulement à des paroles. Les personnages - souvent collectifs - sont avant tout saisis par l'admiration à l'écoute d'un discours: ressort politique sur lequel joue l'orateur, comme le montrent clairement les discours de Brutus et d'Antoine.

(20) $B C \mathrm{~V}, 54.229$

(21) BC II, 142. 592; BC II, 146, 613-614; BC V, 54. 229.

(22) It. (fragmenta) V. 3-5.

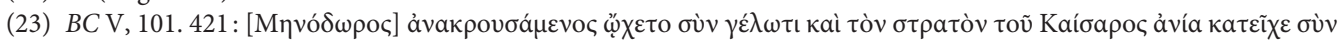

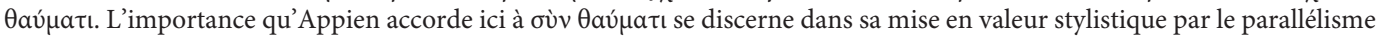

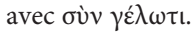

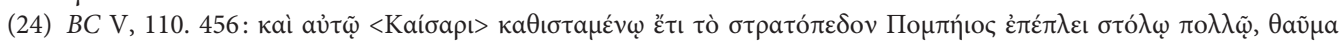

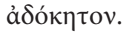

(25) BC I, 16. 67; Ill. 15. 44.

(26) $B C$ II, 79. 333: à Pharsale, les troupes alliées du camp de Pompée contemplent ébahies le bon ordre des troupes

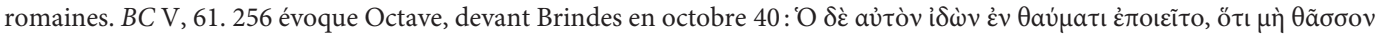

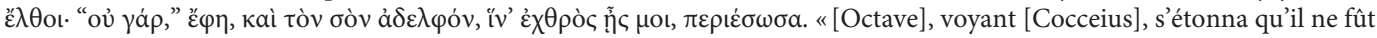
pas venu plus vite, "car, dit-il, si j’ai sauvé ton frère, ce n’est pas pour que tu sois contre moi"». 
L'expression atténuée d'un étonnement face aux faits relatés s'effectue également chez Appien en soulignant la façon dont ils contredisent l'attente logique, dépassent l'entendement ordinaire. Tel est le sentiment éprouvé par les Romains - et par Appien narrateur - après les victoires si vite remportées par Pompée sur les pirates:

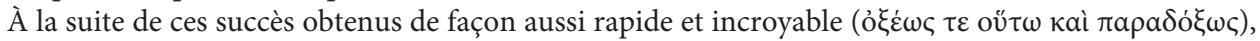
les Romains, portant aux nues Pompée qui se trouvait encore du côté de la Cilicie, le nommèrent commandant de la guerre contre Mithridate avec les mêmes pouvoirs ${ }^{27} \ldots$

Le narrateur commente de la même façon le revirement des troupes de César devant Dyrrachium après une bataille où elles ont cédé à la panique:

Un dieu les poussa au remords et ils eurent honte de leur faute et comme César la leur reprochait sans rudesse et leur accordait son pardon, ils en furent encore plus irrités contre eux-mêmes et, dans

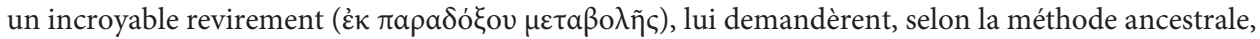
de tirer au sort un homme sur dix pour l'exécuter ${ }^{28}$.

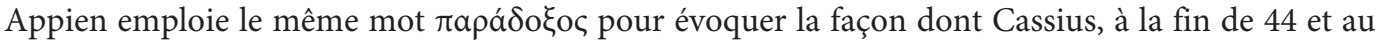
début de 43, réussit en Orient à se rendre maître de douze légions ${ }^{29}$.

Ces quelques exemples, que l'on pourrait multiplier, suffisent à montrer que $\pi \alpha \rho a ́ \delta o \xi o \varsigma$ s'applique toujours à des situations qui contredisent la norme habituelle de pensée par rapport aux situations. La guerre contre les pirates traine en longueur depuis longtemps et plusieurs expéditions importantes contre eux se sont terminées par des échecs; la victoire rapide de Pompée est donc «incroyable» ou «inconcevable». Que des soldats qui, la veille, ont cédé à la panique et voient malgré cela leur illustre général les traiter de façon indulgente, réclament d'être décimés, est «incroyable» par rapport à ce qui a précédé. De même les succès de Cassius en Orient ont de quoi paraître «invraisemblables» quand on en prend connaissance à Rome. Toutefois, ils ne le sont pas pour le lecteur qui a suivi le récit d'Appien et sait par quelle suite d'heureux stratagèmes

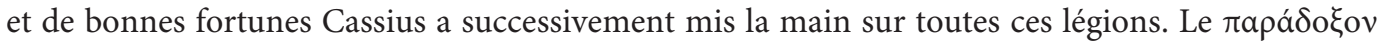
est donc l'impression que ressent celui qui n'est pas au courant de l'enchaînement des événements. C'est aussi pourquoi il peut faire l'objet d'un calcul tactique comme celui que Scipion, le premier Africain, expose à ses officiers, face à une attaque inattendue de Syphax:

$<$ Les ennemis > seront atterrés par la surprise que constituera cette attaque et par le caractère

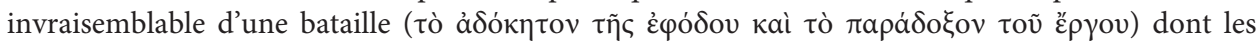
moins nombreux prennent l'initiative; et nous, notre armée ne sera pas répartie en de nombreux points, nous la mènerons regroupée non pas contre la totalité des ennemis, mais contre ceux que nous aurons sélectionnés en prioritée ${ }^{30}$.

L'expression $\pi \alpha \rho \grave{\alpha} \delta$ ó́av présente un sens plus proche de "contre l'attente» - qui déjoue ce qu'on est fondé à attendre d'après le contexte particulier. Trois exemples suffiront à l'illustrer. Le premier évoque le conflit qui, en 87 av. J.-C., oppose les consuls Cinna et Octavius; Cinna a occupé

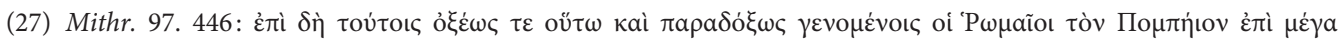

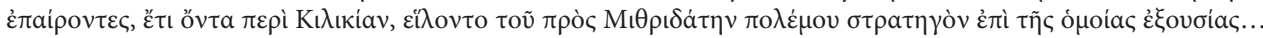

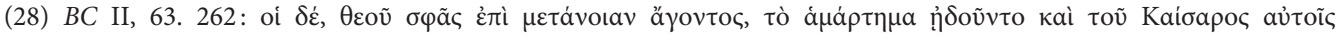

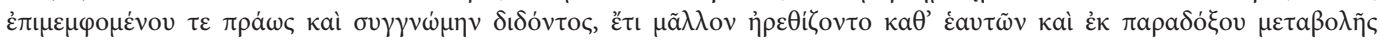

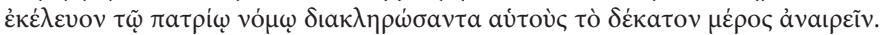

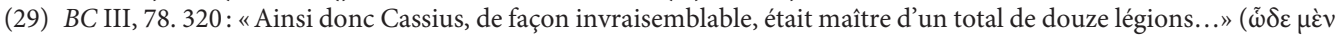

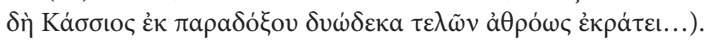

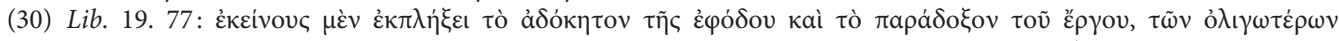

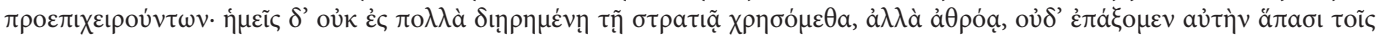
$\dot{\varepsilon} \chi \theta \rho$ oĩৎ, $\dot{\alpha} \lambda \lambda^{\prime}$ oĩ 
le forum avec ses partisans et espère bien faire adopter une loi intégrant les nouveaux citoyens à toutes les tribus:

Cinna se sentait fort de la masse des nouveaux citoyens et s'attendait à passer en force, mais voyant, contre toute attente ( $\pi \alpha \rho a ̀ ~ \delta o ́ \xi \alpha v)$, l'entreprise audacieuse de la minorité l'emporter, il se mit à courir à travers la ville en promettant la liberté aux esclaves ${ }^{31}$.

Un autre exemple est fourni par le passage où Appien évoque l'affolement à Rome quand César, en 49 av. J.-C., après avoir franchi le Rubicon, s'avance rapidement vers la ville:

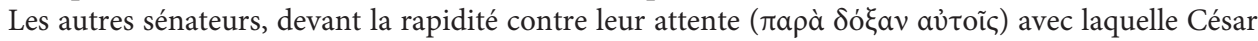
avançait, furent saisis de panique, car ils n'étaient pas encore préparés, et dans leur sidération ( $\sigma \grave{v} v$ $\left.\dot{\varepsilon} \kappa \pi \lambda \eta_{\eta} \xi \varepsilon 1\right)$, ils regrettaient de ne pas avoir accepté les propositions de César ${ }^{32} \ldots$

Le dernier exemple est emprunté à un fragment autobiographique où Appien raconte comment il a échappé à ses poursuivants alors qu'il s'était égaré en Arabie Pétrée lors de la révolte juive de 115-117. Son guide arabe entend le croassement d'une corneille; la première fois, il l'interprète comme signifiant qu'ils se sont égarés, la seconde fois, qu'ils se sont complètement égarés, mais, la troisième, que c'est une chance qu'ils se soient égarés. Effectivement, ils parviennent à une rivière

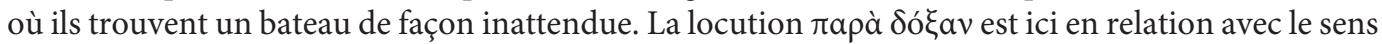
de la vue, ce qui, on l'a déjà noté, est rare concernant l'étonnement.

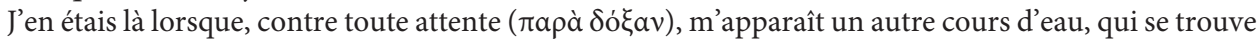
très proche de Péluse, et une trière voguant vers Péluse: j’y montai et je fus sauvé, mais le bateau qui m'attendait sur l'autre cours d'eau fut capturé par les Juifs. De quelle chance j'ai bénéficié et quel a

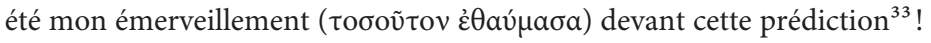

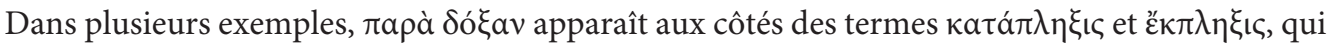
expriment une réaction de stupéfaction paralysante face à un événement inattendu.

\section{L’EMPLOI DES MOTS КАTAП}

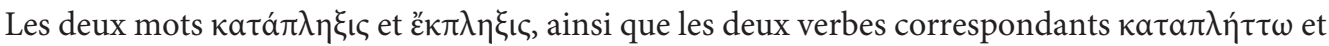
$\dot{\varepsilon} \kappa \pi \lambda \eta ́ \tau \tau \omega$, sont de sens équivalent. Tous deux suggèrent un choc psychologique violent provoquant

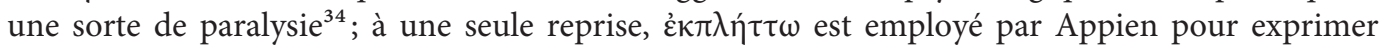
une joie intense après une issue heureuse inattendue ${ }^{35}$. Ailleurs, les deux termes caractérisent la réaction à une très mauvaise surprise. Ils apparaissent associés à d'autres mots évoquant l'inattendu

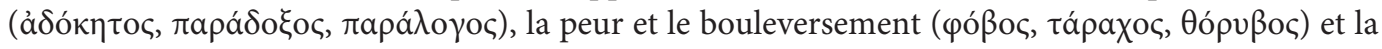

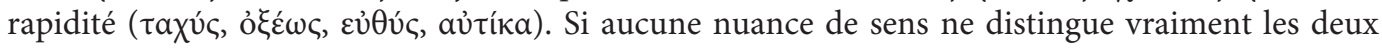

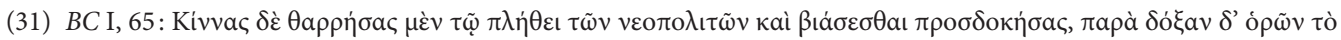

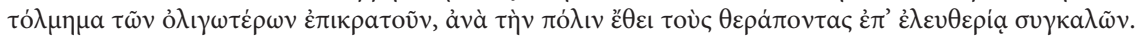

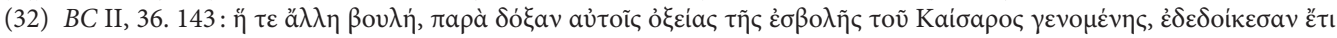

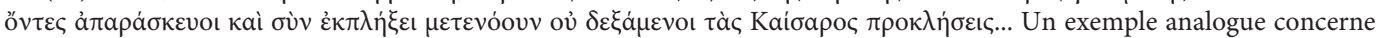
encore César affrontant au Champ de Mars la mutinerie de ses troupes, BC II, 93. 390: «Lui, contre l'attente générale,

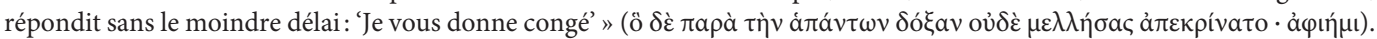

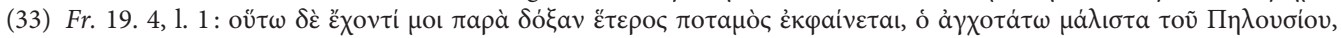

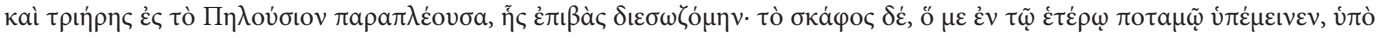

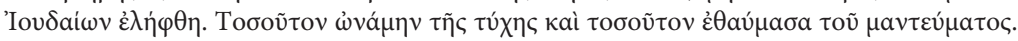

(34) Farrington 2016 a montré son importance dans la tragédie.

(35) Mithr. 116. 566 évoque le retour d'Orient de Pompée, dont les Romains redoutaient l'arrogance après sa victoire sur les pirates en 67: «Lui-même descendit à Éphèse, fit voile pour l'Italie et se hâta de gagner Rome, après avoir, à Brindes,

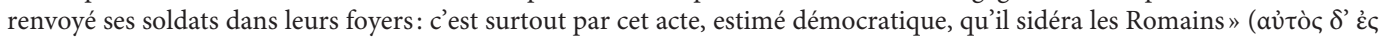
”Е

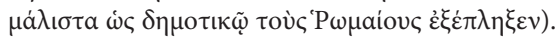




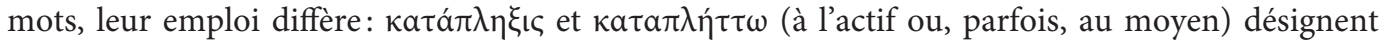
le plus fréquemment une action exercée par le sujet, qui cherche à provoquer la stupéfaction

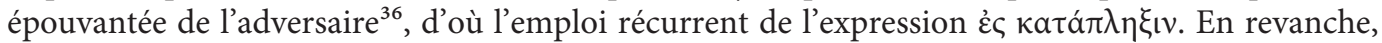
$\varepsilon \ddot{\kappa} \kappa \lambda \eta \eta \xi$ Iৎ et $\dot{\varepsilon} \kappa \pi \lambda \eta \dot{\tau} \tau \omega$ désignent assez souvent plutôt la réaction de celui qui est surpris. Ils peuvent, selon les contextes, être traduits par «stupeur, hébétement, terreur, sidération» et les participes passifs correspondants par «stupéfait, abasourdi, sidéré, tétanisé, terrorisé».

En conséquence, provoquer une telle stupeur chez l'ennemi est un élément essentiel dans l'art de la guerre et Appien montre comment les plus grands stratèges le mettent en œuvre ${ }^{37}$ : Brennus non seulement refuse de recevoir les ambassadeurs de Rome, mais il choisit d'envoyer aux Romains comme ambassadeurs des hommes d'une taille exceptionnelle, à seule fin, écrit Appien, de «les

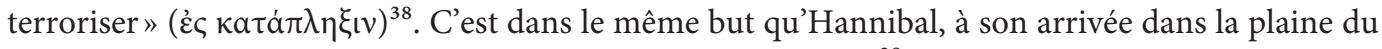
Pô, fait exécuter les prisonniers de la ville gauloise de Taurasia ${ }^{39}$.

Mais c'est sans doute César qui, dans son comportement militaire, use le plus souvent de l'effet de surprise provoquant stupeur et terreur. Ainsi, au moment où il s'avance sur Rome après avoir franchi le Rubicon:

Il envoya partout des messagers à son armée, mais, comme il privilégiait toujours la sidération créée

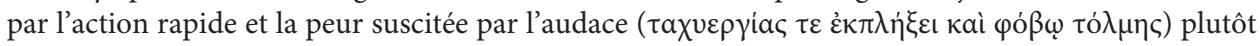
que la force issue des préparatifs, il décida de prendre l'initiative de l'attaque dans une guerre si importante avec seulement ses cinq mille hommes et de s'emparer le premier des points stratégiques de l'Italie ${ }^{40}$.

Ce stratagème s'avère tout à fait opérant:

Il se produisit fuites et migrations de toutes les villes, car on était sous le coup de la stupeur ( $\dot{\omega} \varsigma \grave{\varepsilon} v$ $\dot{\varepsilon} \kappa \pi \lambda \eta \eta \xi \xi \varepsilon l$, et ce fut un exode désordonné accompagné de lamentations: on ignorait la réalité exacte et on pensait que César avançait en force avec une immense armée ${ }^{41}$.

Sur ce point, Appien oppose explicitement César à Pompée, qui avait pourtant, lors de la guerre contre les pirates, créé la stupeur en exploitant lui aussi les vertus de la rapiditée ${ }^{42}$ :

Tandis que Pompée s'était de la sorte révélé si négligent de l'avenir, César, comme je l'ai déjà dit, se précipita sur Brindes aux alentours du solstice d'hiver, comptant par son arrivée inattendue provoquer la plus grande stupeur ( $\mu a \dot{\lambda}\lrcorner \sigma \tau \alpha \dot{\varepsilon} \kappa \pi \lambda \dot{\eta} \xi \varepsilon เ v$ ) chez ses ennemis ${ }^{43}$.

Appien souligne par ailleurs que des individus à l'esprit solide ou des soldats expérimentés

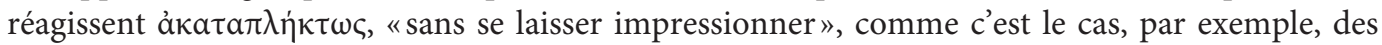

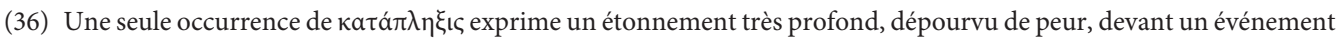
aussi inattendu que l'abdication de Sylla $(B C \mathrm{I}, 3,11)$ : «Si grands étaient encore chez ceux qui le voyaient la crainte de son pouvoir ou le choc provoqué par son abdication, le respect inspiré par sa promesse de rendre des comptes, ou encore une

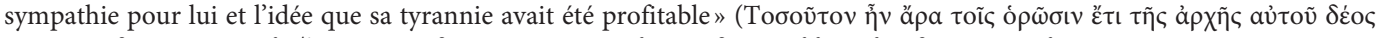

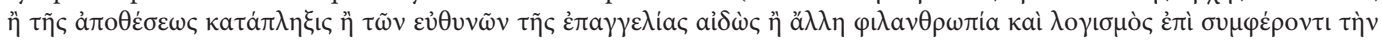

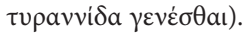

(37) Goldmann 1988, p. 63-64.

(38) Celt. (fr.) 3. 1.

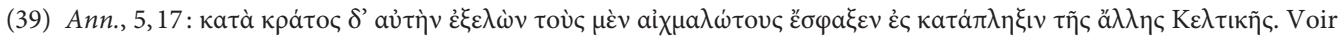
également le comportement de Sylla en BC I, 101. 473.

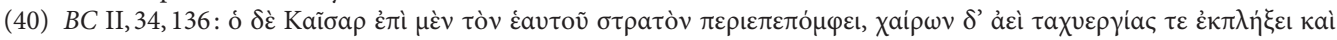

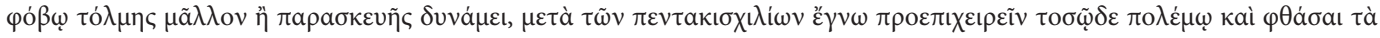

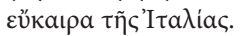

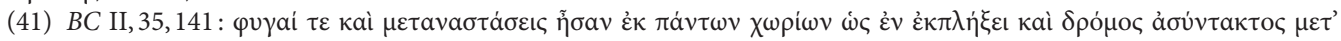

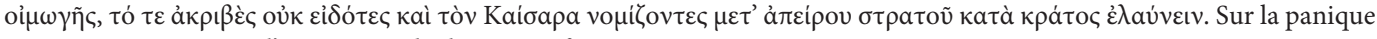
provoquée à Rome par l'avance rapide de César, cf. aussi BC II, 36, 143 cité supra n. 32.

(42) Cf. Mithtr. 97, 446, cité supra n. 27.

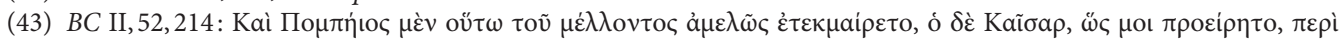

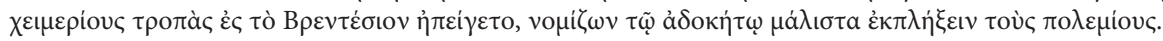


Rhodiens ${ }^{44}$. Cette qualité caractérise aussi bien César que ses assassins ${ }^{45}$. À l'inverse, certaines troupes alliées, en particulier asiatiques, sont, à Pharsale, également saisies de stupeur devant la discipline des troupes romaines:

Les alliés, eux, comme s'ils avaient été des spectateurs de l'affrontement guerrier, restaient hébétés

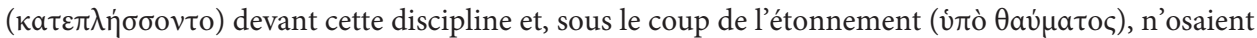
pas aller s'en prendre aux tentes de César, pourtant seulement gardées par un petit nombre de soldats assez âgés, ni courir autour d'elles, ni rien faire d'autre que rester plantés dans leur stupeur ${ }^{46}$.

Ce dernier exemple (où se trouve un emploi exceptionnel chez Appien de $\kappa a \tau a \pi \lambda \eta ́ \tau \tau \omega$ au passif) montre à lui seul l'importance de l'étonnement comme réaction psychologique, avec l'emploi de

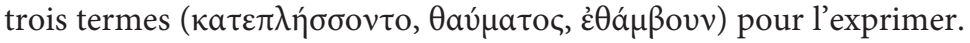

Sauf dans les derniers passages cités, la vue et l'ouïe sont assez rarement mentionnées comme causes de cet étonnement effrayé. En voici toutefois quelques occurrences, la première dans le récit du siège de Numance:

Tout avait de la sorte été minutieusement disposé par Scipion. Les Numantins attaquaient souvent les garnisons, tantôt ici, tantôt là, à tour de rôle, mais ce qu'ils voyaient des défenseurs produisait

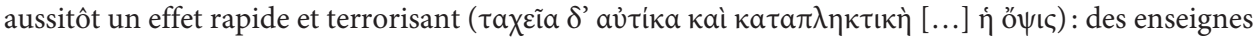
portées haut se levaient de toutes parts, des messagers passaient en courant, les défenseurs des remparts se précipitaient en masse pour monter sur les remparts tandis que des trompettes, sur chaque tour, les encourageaient ${ }^{47} \ldots$

Le phénomène apparaît encore lorsque Appien montre comment Carthage assiégée a réussi en secret à aménager un chenal de sortie et à reconstruire une flotte:

Quand tout fut prêt, les Carthaginois ouvrirent le chenal vers l'aube et sortirent avec cinquante trières et quantité de vaisseaux légers, de brigantins et d'autres bateaux plus petits, et ils s'étaient équipés de façon effrayante pour provoquer la stupeur ( $\dot{\varepsilon} \varsigma \alpha \alpha \dot{\alpha} \tau \lambda \lambda \eta \xi ı)$. Les Romains, devant ce chenal soudain ouvert, avec cette flotte s'ajoutant au chenal, éprouvèrent une si grande stupeur que ${ }^{48} \ldots$

Nous citerons ensuite le moment où, avant le meurtre de César, les conjurés voient Laenas s'adresser longuement à lui:

Aussitôt la vue de ce qui se passait, puis la longueur de la conversation, les frappa de terreur ( $\alpha \tau \dot{\varepsilon} \pi \lambda \eta \sigma \sigma \varepsilon)$ et ils s'entendaient par signes pour se suicider avant d'être arrêtés ${ }^{49} \ldots$

(44) Mithr. 26, 104: «Comme les Rhodiens, sans se laisser décontenancer, avaient répondu par une autre clameur guerrière et s'étaient précipités en masse sur les remparts, les hommes du roi n'entreprirent rien pendant la nuit, et de jour ils

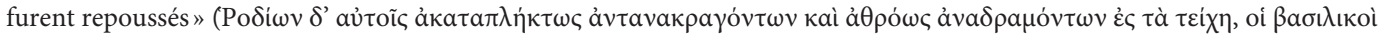

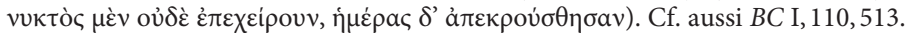

(45) $B C$ II, 150,631 (comparaison entre Alexandre et César): "César lui-même ne se laissait jamais démonter et il

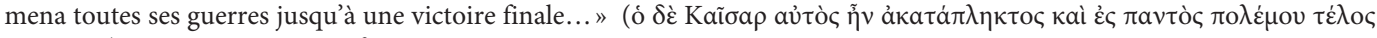

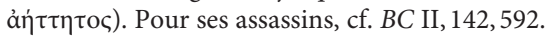

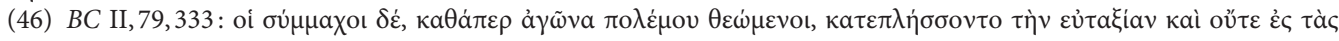

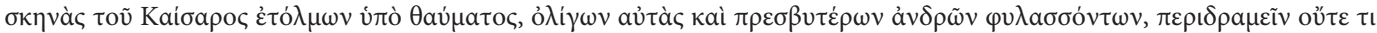

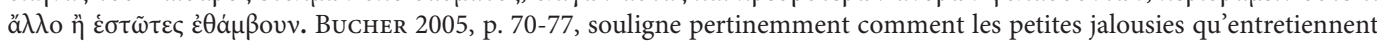
entre eux les peuples de l'Empire, ici celles d'un Alexandrin méprisant les Orientaux, influencent Appien dans plusieurs récits de bataille, et en particulier celle de Pharsale.

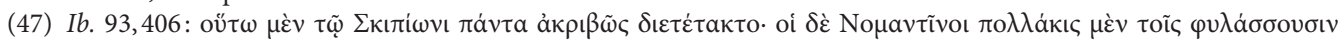

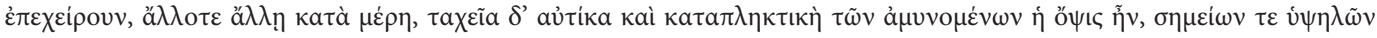

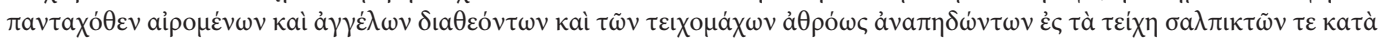

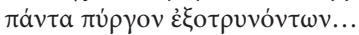

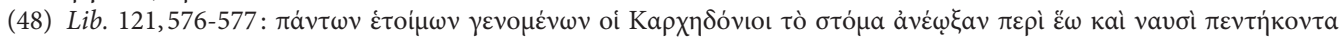

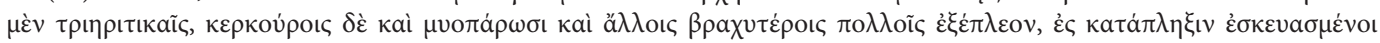


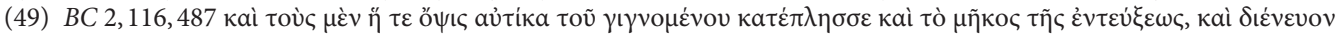

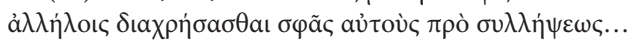


Un dernier exemple, c'est lorsque Appien évoque l'armée d'Antiochos avant la bataille de Magnésie en 190 av. J.-C.:

Le spectacle était comme celui de deux armées dont l'une commencait le combat et l'autre se tenait en réserve: chacune d'elles, pour produire une impression terrorisante ( $\dot{\varepsilon} \varsigma \kappa a \tau \dot{\alpha} \pi \lambda \eta \xi \xi v)$, était redoutablement préparée, par le nombre et l'équipement ${ }^{50}$.

À ces exemples, on peut ajouter plusieurs passages, concernant tous les Gaulois, où Appien souligne l'effet terrorisant que produit leur grande taille ${ }^{51}$.

Dans de plus rares cas, l'étonnement terrorisant est produit par le son, comme lorsque Scipion, le premier Africain, lance une attaque nocturne contre le camp d'Asdrubal:

Alors, poussant une clameur confuse et faisant retentir ensemble trompettes et buccins pour provoquer la terreur ( $\dot{\varepsilon} \varsigma \kappa \alpha \tau \dot{\alpha} \pi \lambda \eta \xi i v)$, ils chassèrent les gardes de leurs postes, comblèrent le fossé et arrachèrent les palissades [...] et les Africains, terrorisés ( $\mu \varepsilon \tau ’ \varepsilon ๋ \kappa \pi \lambda \eta ́ \xi \varepsilon \omega \varsigma)$, bondissaient, tirés $d u$ sommeil ${ }^{52} \ldots$

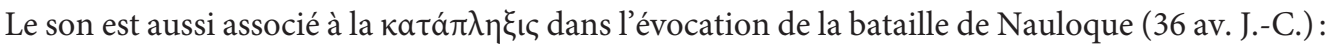
Une fois effectuées les exhortations qui convenaient et les enseignes hissées sur chaque navire, ils s'élancèrent les uns contre les autres, les uns de face, les autres en mouvement tournant, dans les cris,

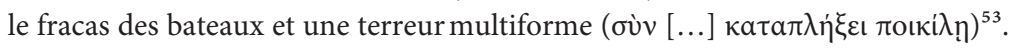

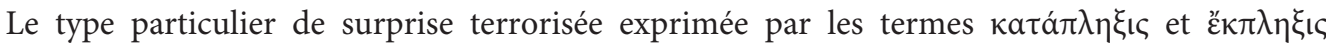
constitue un ressort psychologique essentiel dans les récits d'Appien, dominés par les rapports de force que les personnages et les peuples cherchent à établir entre eux: pour l'emporter, le plus important est de provoquer la peur chez l'adversaire et elle sera d'autant plus grande qu'elle sera accompagnée par la vitesse et la surprise.

\section{LES PHÉNOMÈNES EXTRAORDINAIRES}

Le $\theta$ aṽ $\mu \alpha$ hérodotéen portait souvent sur des phénomènes extraordinaires. Le nombre de ce type de phénomènes mentionnés par Appien est relativement peu élevé, surtout si on le compare aux énumérations régulières auxquelles procède Dion Cassius ${ }^{54}$. Ils ne sont jamais non plus nommés

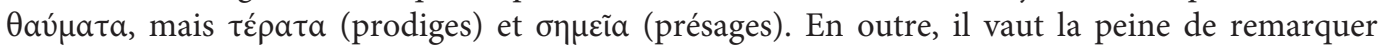
qu'ils ne sont jamais présentés par Appien comme des objets d'étonnement: les mots apparentés à $\theta a \tilde{u} \mu \alpha$ ne sont jamais utilisés pour les introduire ou décrire les réactions qu'ils engendrent. Leur densité est variable d'un livre à l'autre et même à l'intérieur d'un même livre. Rappelons avec V. Rosenberger $^{55}$ que, chez les Romains, les prodiges sont limités à certains types bien catalogués d'événements (qui ne comportent pas les «miracles» auxquels nous ont habitués les traditions bibliques et chrétiennes ni, par exemple, des apparitions de dieux) et que seul le sénat est habilité à les homologuer. Appien ne s'éloigne pas de leur typologie classique: phénomènes qui contredisent l'ordre naturel; phénomènes naturels à valeur de présages.

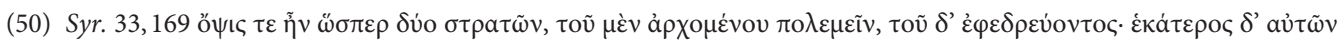

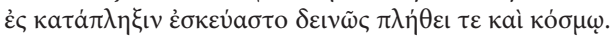

(51) Celt. III, 1; Syr. 6,22.

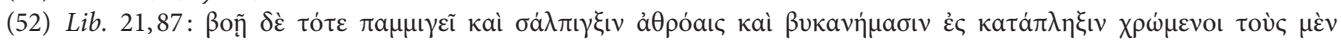

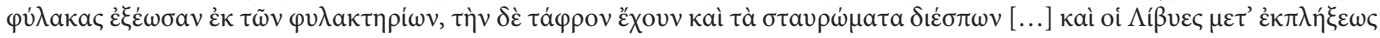

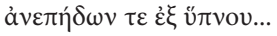

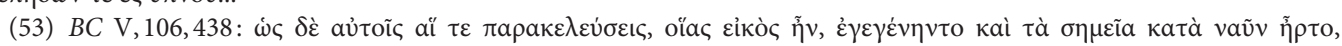

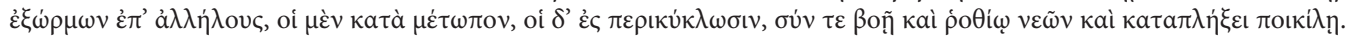

(54) Cf. KuHN-Chen 2002, p. 213-215, qui fournit de nombreuses références.

(55) Rosenberger 1988, p. 25-27. 
Appien ne cite que peu de phénomènes qui contredisent la nature. Pendant que les triumvirs négocient près de Bologne, à Rome, divers phénomènes surnaturels surviennent ${ }^{56}$ : une vache parle une langue humaine, un enfant se met à parler dès sa naissance, des statues se mettent à suer (parfois le sang) ${ }^{57}$, on entend, sans rien voir, des bruits de bataille (cris d'hommes, armes qui s'entrechoquent, chevaux qui courent), il se produit des pluies de pierres ${ }^{58}$, des loups traversent le forum ${ }^{59}$. Appien y ajoute des phénomènes moins nettement antinaturels: chiens qui hurlent comme des loups, «beaucoup de mauvais présages relatifs au soleil ${ }^{60}$, foudre qui s'abat sur les sanctuaires et les statues ${ }^{61}$. Dans les Guerres civiles, quelques autres présages extraordinaires apparaissent: une mule qui met bas ${ }^{62}$, la foudre tombant sur des statues ou des temples ${ }^{63}$. Tout cela constitue un corpus plutôt limité.

Un peu plus fréquents sont les phénomènes naturels à valeur de présages: à Philippes, avant la première bataille, Cassius purifie son armée quand un licteur lui pose une couronne à l'envers, une statue de la Victoire, qu'il avait offerte, tombe; des vols d'oiseaux descendent sur son camp sans pousser le moindre cri, des abeilles s'installent dans son camp ${ }^{64}$; avant la seconde bataille, deux aigles s'affrontent dans l'espace entre les deux armées et celui qui se trouve du côté de Brutus prend la fuite ${ }^{65}$. Brutus, la nuit précédente, aperçoit son «mauvais génie " ${ }^{66}$. Quand l'armée sort du camp, un homme noir ${ }^{67}$ se présente sur son passage et l'armée le massacre. Appien précise qu'elle voit en

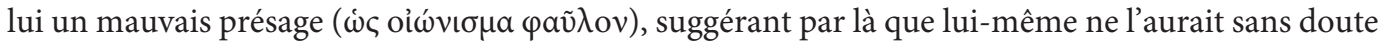
pas interprété ainsi, peut-être habitué qu'il était, en tant qu'Alexandrin, à voir des hommes noirs. La Syriake $e^{68}$ rapporte que, tandis que Séleucos se trouvait en Orient, en Macédoine, une grande flamme surgit du foyer familial sans que personne l'eût allumé et que cela coïncida avec un rêve de sa mère.

Appien présente la plupart des phénomènes extraordinaires qu'il rapporte comme s'étant réellement produits. Les présages aperçus avant Philippes sont présentés par lui comme des signes envoyés par la divinité courroucée contre Brutus et Cassius à cause de l'assassinat de César, leur bienfaiteur ${ }^{69}$. En revanche, les faits extraordinaires concernant Séleucos sont rapportés avec

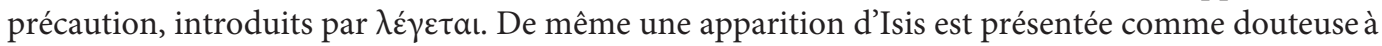

(56) BC IV, 4, 14-15.

(57) Prodige également rapporté en $B C$ II, 36, 144 .

(58) Pluies de sang mentionnées en $B C$ II, 36, 144.

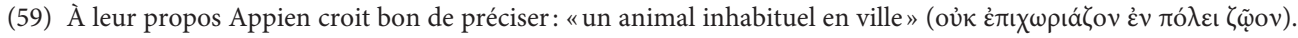

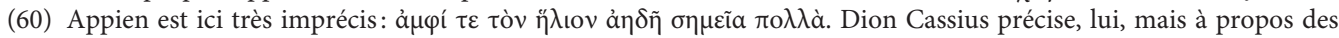
présages précédant Philippes, qu'à Rome, «le soleil tantôt diminuait et devenait tout petit, tantôt se montrait grandi, et triplait même de taille, et un fois, il brilla pendant la nuit» (47,40, tr. E. Bertrand).

(61) Dion Cassius $(47,2.3)$ énumère, à l'occasion de ces mêmes événements, divers présages, mais seuls les bruits d'armes sont communs aux deux auteurs. Selon Dion Cassius, les enseignes de la légion gardant la ville se couvrent de toiles d'araignées, des armes sortant de terre montent au ciel avec fracas, des abeilles se rassemblent au sommet du temple d'Esculape, des vautours se posent sur les temples du Génie du peuple romain et de la Concorde. Julius Obsequens (69) mentionne également les bruits d'armes et la foudre, mais les rattache plutôt à la période qui précède la bataille de Modène (avril 43).

(62) $B C$ I, 83, 377; BC II, 36, 144

(63) $B C$ II et $B C$ IV , 4, 14-15.

(64) $B C$ IV $, 134,563$.

(65) BC IV, 128, 532.

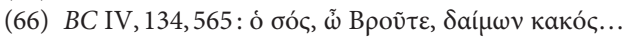

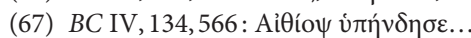

(68) Syr. 56, 284.

(69) $B C$ IV $, 134,563$ : «C'est aussi pourquoi la divinité, bien sûr, s'indigna contre eux et leur en envoya souvent des

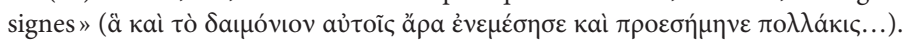


l'aide de $\varepsilon ̋ \delta \xi_{\varepsilon} \varepsilon^{70}$. Même des présages concernant l'histoire romaine récente, comme l'évocation des craintes des Romains devant l'arrivée de Sylla, sont présentés avec réserve: «Ils croyaient, écrit Appien, que ces événements leur avaient été aussi signifiés à l'avance par la divinité dans cette guerre $»^{71}$ alors même que deux de ces prodiges, un tremblement de terre qui détruit plusieurs sanctuaires et l'incendie de l'ancien Capitole, sont tout à fait attestés. Ce qu'Appien met en doute n'est pas leur réalité (comme pour la mule qui met bas ou pour la femme qui accouche d'une vipère), mais l'interprétation que les Romains en donnent alors.

Ce bref panorama montre qu'Appien prend au sérieux les présages qu'il mentionne et que, selon lui, ceux qui n'en tiennent pas compte sont victimes de leur incrédulité, comme le rappellent les exemples illustres de César lors des Ides et d'Alexandre près de Babylone ${ }^{72}$.

Appien relate aussi des faits qu'il présente comme des manipulations de la naïveté populaire par de grands personnages: Scipion (le premier Africain) profite de la conviction, ancrée chez les Ibères, qu'il arrive chez eux par la volonté divine et « fait semblant de tout faire en obéissant à la divinité $»^{73}$. Devant Carthagène, constatant qu'une marée favorable va permettre d'accéder à une zone de rempart mal défendue, il s'écrie: «Il est maintenant arrivé, le dieu qui combat à mes côtés ${ }^{74}$ ». Il exploite le succès de l'opération pour laisser entendre que toutes ses actions lui étaient inspirées par la divinité ${ }^{75}$ et pousse la comédie jusqu'à se retirer souvent seul à l'intérieur du Capitole local, comme pour y recevoir les instructions divines ${ }^{76}$, continuant pendant toute la campagne à agir de la sorte $^{77}$. Le second Scipion l'Africain (dit Émilien) profite auprès de ses troupes devant Carthage de la même aura divine que son ancêtre ${ }^{78}$. Annibal n'est pas en reste pour manipuler l'opinion et, par exemple, se faire passer pour un personnage divin aux yeux des Gaulois de Cisalpine: pour cela, il se contente de changer sans cesse de tenue et d'aspect ${ }^{79}$. Appien nous montre en même temps tous ces grands généraux calculant tout à fait rationnellement leurs actions sans attendre d'inspiration divine. Il décrit toutefois Sertorius comme attaché à « une biche blanche apprivoisée qu'il laissait en liberté et, quand elle disparaissait, il y voyait un mauvais signe pour lui ${ }^{80}$. Quand elle reparait, il reprend ses attaques contre l'ennemi. De tous les auteurs antiques qui ont évoqué cet épisode, Appien est le seul à ne pas présenter l'attitude de Sertorius comme une manipulation ${ }^{81}$. Selon F. Hinard, Appien montrerait par là un Sertorius qui se serait barbarisé ${ }^{82}$.

Parmi les phénomènes extraordinaires susceptibles d'influer sur le cours des choses, il convient de mentionner aussi les rêves: Appien en rapporte plusieurs qui constituent des présages. La mère de Séleucos rêve qu'elle doit trouver un bijou, le lui donner, et qu'il régnera là où l'anneau tombera.

(70) Mithr. 27, 105: «La machine se mit à se disloquer sous son propre poids et il sembla qu'une apparition d'Isis jetait

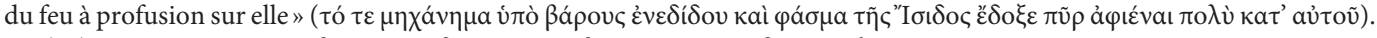

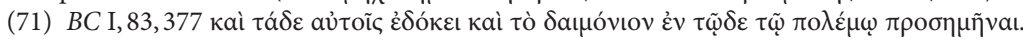

(72) BC II, 149, 619-620 (César); BC II, 152 et 153 (Alexandre et César). BC II, 152, 636: «Ils étaient chacun dédaigneux

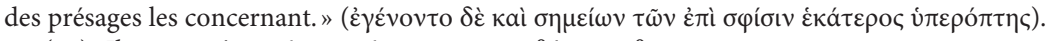

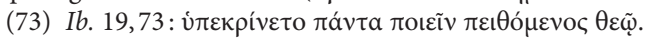

(74) Ib. 21,83.

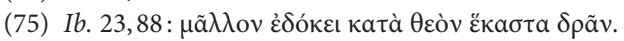

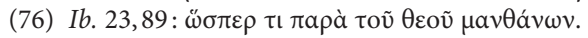

(77) Valère Maxime (I, 2, 2) donne lui aussi cette attitude comme exemple de simulata religio.

(78) Lib. 104,491 et 109, 517.

(79) Ann. 6,21: «Les Gaulois étaient stupéfaits et croyaient qu'il participait d'une nature plus proche de la divinité»

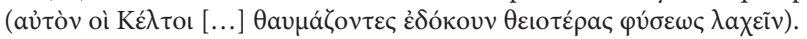

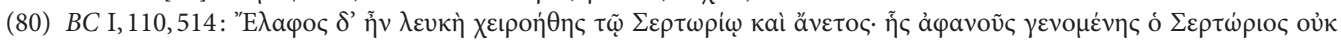

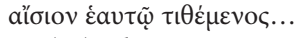

(81) Plut., Sertorius 11,3-8 et 20; Gell., XV,22, 3-5; Frontin, Strat. I, 11, 13; Pline, NH 8, 117; Polyen, 8, 22.

(82) BC I, CUF, 2008 (P. Goukowsky, F. Hinard), n. 633. 
L'anneau tombe dans l'Euphrate et Séleucos devient roi de Babylone ${ }^{83}$. Dans la nuit précédant l'assassinat de César, sa femme Calpurnia le voit en rêve tout ensanglanté et tente de l'empêcher de se rendre au sénat ${ }^{84}$. César, pendant la campagne d'Afrique, aperçoit en rêve une immense armée en larmes, ce qui lui rappelle qu'il doit fonder une colonie à Carthage ${ }^{85}$. C'est à la suite d'un songe que Didon découvre que le tyran de Tyr, Pygmalion, a tué son époux, et qu'elle quitte Tyr pour fonder Carthage ${ }^{86}$. Antigonos, qui gouvernait la Syrie, voit en rêve un Mithridate de son entourage moissonner l'or qu'il avait semé et se retirer dans le Pont ${ }^{87}$. Le fameux Mithridate VI fut amené par un songe à ne pas couper, comme il l'avait prévu, le bois sacré consacré à Lèto pour construire des machines de guerre ${ }^{88}$. Un rêve amène également Lucullus à accorder la liberté à la ville de Sinope, qu'il vient d'assiéger ${ }^{89}$. Pompée, la veille de Pharsale, voit en rêve qu'un temple est consacré à Rome à Vénus Victrix; ignorant que César vient de faire le vœu de lui en bâtir un s'il est victorieux, l'entourage de Pompée y voit un présage de victoire ${ }^{90}$. Deux rêves indiquent à Sylla, l'un la ville $\mathrm{d}^{\prime}$ Asie où il doit offrir une hache à Vénus ${ }^{91}$, l'autre l'approche de sa mort ${ }^{92}$. Enfin, à Philippes, un rêve prescrit à Octavien de ne pas rester à l'intérieur de son camp, qui sera effectivement pris par les soldats de Brutus ${ }^{93}$.

Ces passages permettent de conclure que tous les rêves relatés par Appien sont pour lui d'authentiques présages qui, tous, se sont révélés justes. Il est donc implicitement recommandé de tenir compte des conseils ou des avertissements qu'ils contiennent. Les idées d'Appien en la matière n'ont rien d'original à son époque ${ }^{94}$ et ne montrent pas en lui un esprit qui serait particulièrement porté vers l'irrationnel.

Si Appien montre que certains généraux se permettent de simuler l'inspiration divine, il mentionne par ailleurs fréquemment des interventions de la divinité qu'il présente comme véritables: nous renvoyons sur ce point à celles qu'a cataloguées B. Goldmann ${ }^{95}$. Il est immédiatement à noter qu'il s'agit toujours d'un $\theta \varepsilon o ́ \varsigma$ ou $\delta a \mu$ óvı Appien mentionne la $v \varepsilon ́ \mu \varepsilon \sigma ı \varsigma$, il ne la présente pas comme une divinité, mais plutôt comme l'action vengeresse de la divinité, dont il faut se garder, comme le conseille au sénat un ami de Scipion après la défaite de Carthage dans la deuxième guerre Punique: «Il est bon, au milieu des succès, de se garder de la vengeance des dieux et de la jalousie des hommes ${ }^{97}$. Appien emploie volontiers le verbe $v \varepsilon \mu \varepsilon \sigma a ́ \omega$, comme lorsque les Romains décident de mener une guerre sans déclaration contre les Samnites ( Un dieu châtia leurs propos orgueilleux ${ }^{98}$ ) ou, plus curieusement, quand Octave

(83) Syr. 56, 284.

(84) $B C$ II, 115,480 .

(85) Lib. 136, 645.

(86) Lib. 1,2.

(87) Mithr. 9,27.

(88) Mithr. 37, 106.

(89) Mithr. 83, 370.

(90) BC II, 69, 284.

(91) BC I, 97, 455.

(92) BC I, 105, 492.

(93) BC IV , 110, 463.

(94) Cf. del Corno 1978; Hopfner 1937 (l'auteur montre que, dans l'Antiquité gréco-romaine, seuls Carnéade et les épicuriens mettent en doute la valeur prémonitoire des rêves).

(95) Goldmann 1988, p. 27-29.

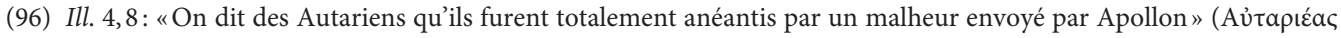

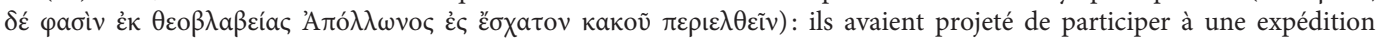
gauloise contre Delphes. Cf. Goldmann, 1988, p. 24.

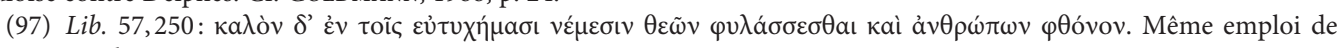

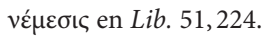

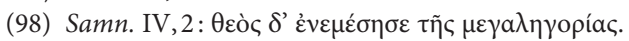


vient de l'emporter successivement sur Sextus Pompée puis sur Lépide, la divinité «se montra jalouse de ses succès et son armée se mutina ${ }^{99}$.

Cette action vengeresse s'exerce en particulier dans trois cas:

- quand une promesse, un engagement n'est pas tenu, ou qu'un traité est violé. Quand les Gaulois Sénons, rompant leur accord avec Rome en devenant mercenaires chez les Étrusques, massacrent en outre les ambassadeurs romains, ils finissent défaits, massacrés ou réduits en esclavage, et l'historien commente: «Tel fut le châtiment des Sénons pour leur forfait contre des ambassadeurs ${ }^{100}$. En 113 av. J.-C., un consul romain se comporte de façon déloyale à l'égard des Teutons et il est «puni de sa déloyauté en perdant beaucoup d'hommes ${ }^{101}$. Mais les violations de traités, les scélératesses accompagnées d'atrocités commises en Ibérie par les consuls Lucullus et le préteur Galba restent impunies des dieux comme des hommes ${ }^{102}$. Certes, c'est un rescapé des massacres de Lusitanie, Viriathe, qui mène ensuite une révolte de plusieurs années (147-139) contre les Romains ${ }^{103}$, mais Appien ne montre pas là une vengeance divine. Quand Viriathe est assassiné traîtreusement sur ordre du consul Servilius Caepio, la divinité n'intervient pas non plus pour punir ce forfait ${ }^{104}$.

- quand une guerre est engagée sans déclaration: c'est le cas en Samn.IV,2 cité plus haut. Appien évoque encore un traité conclu sur place avec les Numantins par un général, mais ignoré de son successeur, qui explique ses difficultés en soulignant que «la présente guerre, votée par les Romains en violation de ces traités, a été placée pour eux sous de mauvais auspices ${ }^{105}$; il s'agit là, toutefois, d'une raison invoquée par lui pour sa défense et non d'une affirmation d'Appien.

- quand des sacrilèges sont commis (par exemple, un temple pillé ${ }^{106}$ ).

L'intervention divine s'effectue aussi sous la forme d'une démence envoyée par les dieux $\left(\theta \varepsilon o \beta \lambda a ́ \beta \varepsilon\llcorner\alpha)^{107}\right.$, amenant un individu à prendre de mauvaises décisions ou à ne pas savoir exploiter les circonstances (Pompée, Cassius, Brutus, Sextus Pompée).

La divinité apparaît enfin comme volonté dirigeant l'histoire: elle veut le passage de la République au Principat ${ }^{108}$ et les républicains ont beau prendre toutes les mesures humaines pour remporter la victoire, à Pharsale ou à Philippes, en s'assurant la supériorité numérique, le choix d'un bon site pour leurs camps, une bonne logistique (provisions abondantes, bonnes communications avec l'arrière...), le sort semble s'acharner contre eux et provoquer leur défaite. En même temps, dans les cas de Brutus et de Cassius, la divinité venge le meurtre de César, qu'Appien juge impie ${ }^{109}$.

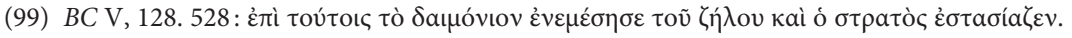

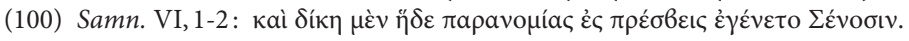

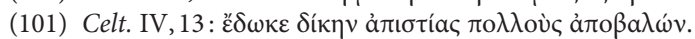

(102) Ib. 52, 218-221: massacre de Cauca par Lucullus, bien que les habitants aient rempli les conditions qu'il exigeait; Ib. 59-60, 249-255: massacre des Lusitaniens par Galba.

(103) Ib. 60,254, puis long récit de la «guerre de Viriathe» de 61 à 75.

(104) $I b .75,321$.

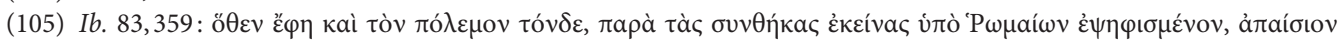

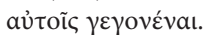

(106) Samn. (fr.)12,4-6 (Pyrrhus, après avoir pillé le temple de Perséphone à Locres voit sa flotte détruite par une tempête et les objets volés dans le temple rapportés sur le rivage par les vagues); Syr. 66, 352 (Antiochos Épiphane meurt de consomption après avoir pillé le temple d'Aphrodite à Élymaïs en Égypte).

(107) Goldmann 1988, p. 33 à 44, traite ce point exhaustivement.

(108) Goldmann 1988, p. 29-31.

(109) Idée soulignée avec insistance par lui dans les dernières lignes de $B C$ II, 154,649, au début et à la fin de $B C$ III (1.1 et 98. 408-409), au début de $B C$ IV (1.1), puis à la fin du long développement qu'en conclusion il consacre à Brutus et Cassius (BC IV, 134, 562-568). 
Le $\theta$ aṽ $\mu \alpha$ ne constitue pas chez Appien une matrice de son entreprise historique. Bien que, comme Hérodote, il procède géographiquement, pays par pays, il ne souligne guère ce qui, dans les comportements locaux, peut être étonnant et ne cherche pas vraiment à l'expliquer. Une telle absence d'intérêt ethnographique est particulièrement frappante dans le Livre ibérique. L'étonnement, sous forme de stupeur paralysante, est en revanche dans ses récits un des ressorts le plus souvent mis en œuvre. C'est un élément décisif dans l'art militaire, où il faut soit le créer chez l'adversaire, soit, pour le bon soldat, s'accoutumer à ne pas le ressentir. Dans les conflits politiques, cela joue également un rôle déterminant, par exemple dans le conflit entre Antoine et Octave. Son Histoire romaine n'est pas néanmoins dépourvue d’interrogations et, comme l'annonce sa Préface, sa problématique se concentre consciemment sur une question historique majeure: comment l'empire romain a-t-il pu atteindre une telle durée et une telle étendue? Appien, dès cette Préface, fournit d'emblée des réponses, qu'illustrera tout son récit, en soulignant les qualités constantes des Romains au milieu des vicissitudes de leur histoire: sagacité, courage, endurance et résistance, auxquelles s'ajoute la capacité à ne pas "se laisser ni exalter par leurs succès avant d'avoir assuré solidement leur victoire, ni abattre par leurs malheurs $»^{110}$ - autant de qualités qui rationalisent le récit de l'ascension de Rome. Une telle démarche laisse peu de place à un étonnement de type hérodotéen. Appien s'est posé des questions avant même de concevoir le projet de son Histoire romaine. Quand il la rédige, il pense posséder les réponses, qu'il expose dans sa Préface et autour desquelles il articule tout son récit. Il existe toutefois un élément que sa narration développe avec insistance sans qu'il soit annoncé ni dans la Préface générale, ni dans la préface aux Guerres civiles qui ouvre leur livre I (1-6): l'absence de scrupules, la cruauté, la mauvaise foi à toute épreuve d'un peuple persuadé d'être toujours dans son bon droit. L'importance de ce que suggère le récit, alors même que les passages plus théoriques n'en disent rien, doit être mise en rapport avec la situation d'Appien et des autres historiens sous l'empire romain: il convient de ne pas oublier qu'ils vivent sous un régime où l'expression est très contrôlée et où certaines positions ne peuvent pas être exprimées directement. Il convient donc de prendre autant en considération ce que souligne un récit d'allure apparemment neutre que les déclarations explicites des auteurs. Chez Appien, la critique implicite des comportements romains au cours de la conquête ou la valorisation de certains héros républicains dans leur lutte contre des fondateurs du principat présentés, eux, sous un jour souvent peu avantageux, ne doit pas nous amener à discerner sans la plus grande prudence des prises de position d'Appien par rapport à son propre temps. Le « régime d'historicité» ${ }^{111} \mathrm{~d}$ 'Appien se distingue en effet de celui d'un Thucydide ou d'un Polybe en ce qu'il n'envisage pas l'étude du passé comme comportant des leçons à tirer pour l'avenir. Appien, contemplant le passé, cherche beaucoup à en expliquer certains aspects, mais il semble figé dans le présent d'un empire romain qui, par son étendue et sa durée, a largement surpassé tous ses prédécesseurs ${ }^{112}$ et constitue comme une perspective indépassable. Il rapporte pourtant les paroles de Scipion Émilien, devant Carthage en flammes, envisageant non sans angoisse que Rome puisse un jour connaitre un sort analogue. Le propos reste toutefois trop général pour qu'on puisse y voir une allusion à un possible

(110) Préf. 11.43: «L'empire des Romains, lui, s’est distingué par son extension et sa durée grâce à leur sagacité et leur bonne fortune et, pour l'acquérir, ils ont surpassé tous les autres par leur courage, leur endurance et leur résistance, sans se laisser ni exalter par leurs succès avant d'avoir assuré solidement leur victoire, ni abattre par leurs malheurs» ( $\tau \grave{\alpha}$

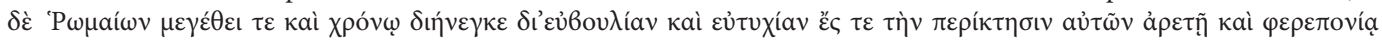

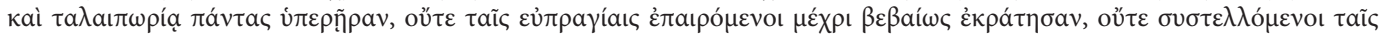
$\sigma u \mu \varphi о \rho а i \varsigma)$. Le mot $\tau \alpha \lambda \alpha \iota \pi \omega \rho i ́ \alpha$ est délicat à traduire: étymologiquement, il signifie le fait de supporter des mutilations, et cela correspond bien à ce que souligne la fin de la phrase.

(111) Notion introduite par F. Hartog 2003 dans son ouvrage éponyme et définie (p. 35) comme «façon d'articuler passé, présent et futur».

(112) Préf. 8, 27. 
déclin de l'empire romain. Par ailleurs, les nostalgies républicaines qu'il est possible de discerner chez lui ${ }^{113}$ ne constituent pas une remise en cause du système impérial sous lequel il vit et dont il est un notable: elles expriment le regret d'un temps héroïque où certains hommes étaient d'un comportement plus noble, mais ne constituent nullement une projection vers l'avenir.

Philippe Torrens Docteur en Histoire Ancienne

\section{Bibliographie}

Bucher, G. S., 2005, «Fictive elements in Appian’s Pharsalus narrative», Phoenix, 59 (1-2), p. 50-76.

del Corno, D., 1978, «I sogni e la loro interpretazione nell'età dell'impero », ANRW II, 16. 2, Berlin-New York, p. 1605-1618.

Famerie, É. (éd.), 1993, Concordantia in Appianum, Hildesheim, Zürich, New York.

Goldmann, B., 1988, Einheitlichkeit und Eigenständigkeit der Historia Romana des Appian, Hildesheim.

Hopfner, H., 1937, «Traumdeutung», RE V, 2, p. 2233-2245.

Hunzinger, Ch., 1995, «La notion de $\theta \tilde{\omega} \mu \alpha$ chez Hérodote», Ktèma, 20, p. 47-70.

Kuhn-Chen, B., 2002, Geschichtskonzeptionen griechischer Historiker im 2. und 3. Jahrhundert n. Christ, Frankfurt am Main.

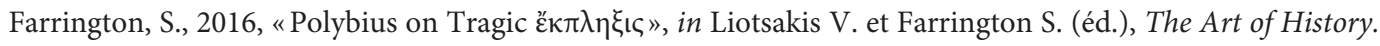
Literary Perspectives on Greek and Roman Historiography, Berlin, Boston, p. 161-168.

Hartog, F., 2003, Régimes d'historicité, Paris.

(113) En particulier dans les dernières pages de $B C$ IV, où il se livre à un éloge vibrant de Brutus et Cassius, avant de les condamner pour le meurtre de César. Cf. notre introduction à ce livre dans la collection «La roue à livres», Paris, 2008, p. 47-48. 